\title{
Different Methods in Diagnosis of Pulmonary and Extrapulmonary Tuberculosis
}

\author{
HEBAT-ALLAH G. RASHED, M.D.*; MOHAMMED Z. ABD EL-RAHMAN, M.D.*; \\ ASMAA O. AHMED, M.D.*; ALAA R. MAHMOUD, M.D.** and AMAL M.H. ALAM EL-DIN, M.Sc.* \\ The Department of Clinical Pathology, Faculty of Medicine, Assiut University*, Assiut, Egypt and \\ The Department of Chest, Kena Faculty of Medicine, South Valley University**, Kena, Egypt
}

\begin{abstract}
Background: Tuberculosis (TB) has been a major health problem in developing countries.

Rapid diagnosis of Mycobacterium tuberculosis infection plays a critical role in controlling the spread of tuberculosis. Conventional methods may take up to several weeks or longer to produce results. This study aimed to compare different methods like culture on Bio F-M media, automated BacT/ ALERT MP bottles, and urine lipoarabinomnnan by ELISA.

Subjects and Methods: This study was done on 100 patients suspected to have tuberculosis, cases divided into two groups: (Group A): 68 clinically suspected pulmonary TB cases. (Group B): 32 clinically suspected extrapulmonary TB cases. All samples from Group A and B were subjected to direct staining by Zeihl-Neelsen stain, culture of the samples on Lowenstein Jensen media, Bio FM media and automated BacT/ALERT MP bottles and detection of urine lipoarabinomannan by ELISA.

Results: In suspected pulmonary tuberculosis cases the sensitivity and specificity of Bio FM were $87.2 \%, 100 \%$ respectively, sensitivity and specificity of BacT/ALERT MP were $74.4 \%, 100 \%$ respectively, sensitivity and specificity of urine lipoarabinomannan were $20.5 \%, 96.9 \%$ respectively and in suspected extrapulmonary tuberculosis cases were the sensitivity and specificity of Bio FM were $87.5 \%, 100 \%$ respectively, sensitivity and specificity of BacT/ALERT MP were $50 \%, 100 \%$ respectively, sensitivity and specificity of urine lipoarabinomannan were $12.5 \%, 100 \%$ respectively.

Conclusion: The sensitivity of both solid (Lowenstein Jensen) and liquid (Bio FM) media were the same and better than BacT/ALERT MP. Bio FM media and BacT/ALERT MP show shorter time than Lowenstein Jensen for detection of mycobacterial growth. Detection of urine lipoarabinomannan by ELISA is insensitive for the diagnosis of TB.
\end{abstract}

Key Words: Tuberculosis - Methods of TB culture - Lipoarabinomannan.

Correspondence to: Dr. Amal M.H. Alam El-Din, E-Mail: amal.alameldin@yahoo.com.

\section{Introduction}

TUBERCULOSIS is an infectious bacterial disease caused by Mycobacterium tuberculosis, which most commonly affects the lungs (pulmonary TB) but can affect other sites as well (extrapulmonary TB). It is estimated that 2 billion of the world's population are latently infected with Mycobacterium tuberculosis resulting in 9.6 million cases of active Tuberculosis (TB) and 1.5 million deaths annually [1].

Conventional method of $\mathrm{ZN}$ staining is routinely performed for diagnosis of tuberculosis as it is rapid and inexpensive method with high specificity. The major disadvantage of this method is its low sensitivity, since more than 10,000 bacilli per $\mathrm{ml}$ sputum are needed for reliable detection [2].

Mycobacterial culture, which is regarded as the diagnostic gold standard, needs 10-100 viable bacilli per ml sputum and is therefore much more sensitive but requires a maximum incubation time of 6-8 weeks [3].

Lowenstein Jensen culture (LJ) is the most widely used in low-income countries, it is an egg based medium developed from Jensen's modification of Lowenstein's formula. The inoculation time of the bacilli is up to 8 weeks. Bio-FM is an enriched Middlebrook 7H9 medium, optimized for rapid mycobacterial growth whose selectivity is enhanced by a selective VCA (Vancomycin, Colistin and Amphotericin B) supplement, containing a colored indicator that allows the detection of positive cultures which turn into a dark blue to violet color. The results are confirmed by microscopy after $\mathrm{ZN}$ staining [4] 
During lasts decades automated systems for detection of growth in different microorganisms in liquid medium were developed. Most automated systems are based on different technologies, such as colorimetric methods that detect bacterial $\mathrm{CO} 2$ production like BacT/ALERT 3D system [5].

As a strategy for rapid TB diagnosis, the detection of Mycobacterium tuberculosis antigens has been explored over several decades. Lipoarabinomannan (LAM), a $17.5 \mathrm{kD}$ glycolipid component of the outer cell wall of mycobacteria is an attractive diagnostic target [6]. LAM is released when Mycobacterium tuberculosis is lysed by the host immune system filtered by the kidneys and can be detected in the urine as a potential same day diagnostic test for tuberculosis [7].

\section{Subjects and Methods}

This study was done on 100 patients suspected to have tuberculosis (68 cases pulmonary and 32 cases extrapulmonary), age of the patients ranged from 15-76 years they included 62 male and 38 female from patients admitted to Chest Department in Assiut University Hospital, outpatient from TB clinic and orthopedic operation room during the period from November 2014 to February 2016.

Cases divided into two groups: (Group A): 68 clinically suspected pulmonary TB cases. (Group B): 32 clinically suspected extrapulmonary TB cases. All patients were subjected to the following: Full history taking, clinical examination and chest $\mathrm{X}$-ray.

Samples: (Pulmonary TB) sputum $(\mathrm{n}=53)$ and bronchoalveolar lavage $(\mathrm{n}=15)$. (Extrapulmonary) different kinds of clinical samples including pleural fluid $(\mathrm{n}=11)$, pus $(\mathrm{n}=6)$, urine $(\mathrm{n}=6)$ and stool $(n=5)$, ascetic fluid $(n=3)$, bone tissue $(n=1)$ were collected.

All samples from Group A and B were subjected to the following:

Microbiological tests: Direct staining by ZeihlNeelsen stain, culture of the samples on Lowenstein Jensen media, Bio FM media and automated MP BacT/ALERT MP bottles.

Specific test: Lipoarabinomannan by ELISA for urine samples from Group A, B and 22 apparently healthy control groups.

Specimens collected from contaminated sites were liquefied, decontaminated and concentrated by using the modified petroff's method but Spec- imens collected from sterile sites were concentrated by centrifugation ( $3000 \mathrm{rpm}$ for $15 \mathrm{~min}$ ) without prior decontamination [8].

\section{Smear preparation:}

Smears were prepared from all samples and examined for the presence of Acid-Fast Bacilli (AFB) using Zeihl-Neelsen stain [9]

Inoculation on Lowenstein Jensen Medium (LJ) "Gold Standard":

Then $0.5 \mathrm{~mL}$ were inoculated in the culture medium (LJ) (bioMérieux, france Ref 42 089) and incubated at $37^{\circ} \mathrm{C}$ for 8 weeks. The readings of cultures were done weekly for 8 weeks.

\section{Inoculation on Bio-FM medium:}

Specimens culturing was done on Bio-FM medium (BIO-RAD, France, Ref 70, 160-70, 161). It is an enriched Middlebrook 7H9 medium, optimized for rapid mycobacterial growth whose selectivity is enhanced by a selective VCA (Vancomycin, Colistin and Amphotericin B) supplement, containing a colored indicator that allows the detection of positive cultures which turn into a dark blue to violet color. The results are confirmed by microscopy after ZN staining. Samples were incubated for 5-6 weeks at $37^{\circ} \mathrm{C}$. Reading cycle was performed by the following way: 2-4 weekly readings for 3-4 weeks, then twice a week for another 2 weeks.

\section{Examination of the sediment and liquid medium:}

- The bottom of the tubes and the liquid medium were carefully examine.

- If signs of growth were present.

- Dark blue/violet grains or small flakes that have settled at the bottom of the tube: Presumption of MTB.

Inoculation in Bottle for BacT/ALERT MP (bioMérieux, france, Ref 43-03064).

The MP bottle contained $10 \mathrm{~mL}$ of liquid medium (7H9 Middebrook) with casein, serum bovine albumin and catalase, $0.5 \mathrm{ml}$ of the digested and decontaminated sample was inculated in a bottle then $0.5 \mathrm{~mL}$ of antibiotic supplement $\mathrm{MB} / \mathrm{BacT}$ (amphotericin B, azlocillin, nalidixic acid, polymyxin B, trimethoprim, vancomycin) was added to reduce the incidence of other bacteria contamination, in case of sterile Specimens $0.5 \mathrm{~mL}$ of reconstitution fluid and $0.5 \mathrm{~mL}$ of samples was inoculated in a bottle and incubated in the BacT/ ALERT 3D system for 4 weeks all samples were identified as positive by the instrument BacT/ 
ALERT 3D the ZN staining was performed to confirm positive results.

\section{Lipoarabinomannan by ELISA:}

Urine sampels were collected in a sterile plastic container and centrifuged for 20-mins at the speed of 2000-3000rpm, and then the supernatant was taken and stored at $-20^{\circ} \mathrm{C}$, until processing. Lipoarabinomannan was measured in urine by ELISA (Human LAM ELISA kit. WKEA, China). The kit uses Purified Human LAM antibody to coat microtiter plate wells, make solid-phase antibody, then added LAM to the wells. Combined LAM antibody with which the enzyme was labeled, becomes the antibody-antigen-enzyme-antibody complex. After washing completely, substrate was added and the substrate becomes blue in color in the HRP enzyme-catalyzed reaction, the reaction is terminated by the addition of a sulphuric acid solution and the color change is measured spectrophotometrically at a wavelength of $450 \mathrm{~nm}$. The concentration of LAM in the samples is then determined by comparing the O.D. of the samples to the O.D. of the control samples, the cutoff point of lipoarabinomannan was at 0.35 .

\section{Data analysis:}

Data were analyzed by computer program SPSS" Version 21. Data expressed as mean \pm SD, frequencies and percentage. Using Lowenstein Jensen culture as a gold standard test, and Bio FM media, urine lipoarabinomannan or BacT/ALERT MP bottle as a screening tests; sensitivity, specificity, positive predictive and negative predictive value were calculated. Based on testing normality for quantitative variables, Mann-Whitney was used to compare mean between two independent groups, A significant $p$-value was considered when it was less than 0.05 .

\section{Results}

This study was done on 100 patient, 68 cases suspected to have pulmonary tuberculosis they include $44(64.7 \%)$ male and $24(35.3 \%)$ female with age ranged from 17 to 77 years with mean age \pm S.D $47.35 \pm 14.58$ years and 32 cases suspected to have extrapulmonary tuberculosis they include $18(56.2 \%)$ male and $14(43.8 \%)$ female with age ranged from 24 to 78 years with mean age \pm S.D $44.81 \pm 15.24$ years.

There was statistically significant differences between the positive LJ groups and positive Bact/ ALERTgroup $\left(p^{1}=0.001\right)$ and between the positive LJ groups and positive Bio FM group $\left(p^{2}=0.007\right)$ but there was no statistically significant differences between positive Bio FM media group and positive BacT/ALERTgroup $\left(p^{3}=0.205\right)$ (Table 4).

Table (1): Positive results among studied cases by different methods.

\begin{tabular}{llllll}
\hline & $\begin{array}{c}\text { Z-N stain } \\
\text { positive } \\
\mathrm{n}(\%)\end{array}$ & $\begin{array}{c}\text { LJ } \\
\text { positive } \\
\mathrm{n}(\%)\end{array}$ & $\begin{array}{c}\text { BLERT } \\
\text { positive } \\
\text { n (\%) }\end{array}$ & $\begin{array}{c}\text { Bio FM } \\
\text { positive } \\
\mathrm{n}(\%)\end{array}$ & $\begin{array}{c}\text { LAM } \\
\text { positive } \\
\mathrm{n}(\%)\end{array}$ \\
\hline - Pulmonary & 40 & 39 & 29 & 34 & 9 \\
$\mathrm{~N}=68$ & $(58.8 \%)$ & $(57.4 \%)$ & $(42.6 \%)$ & $(50 \%)$ & $(13.2 \%)$ \\
- Extrapulmonary & 6 & 8 & 4 & 7 & 1 \\
$\mathrm{~N}=32$ & $(18.8 \%)$ & $(25 \%)$ & $(12.5 \%)$ & $(21.9 \%)$ & $(3.1 \%)$ \\
\hline
\end{tabular}

Table (2): Comparison of the Bio FM media with Lowenstein Jensen.

\begin{tabular}{lllllll}
\hline Bio FM & \multicolumn{2}{c}{$\begin{array}{c}\text { Lowenstein } \\
\text { Jensen }\end{array}$} & $\begin{array}{c}\text { Sensi- Speci- } \\
\text { tivity }\end{array}$ & ficity & PPV & NPV \\
& Positive Negative & & & \\
\hline $\begin{array}{c}\text { Pulmonary: } \\
\text { Positive } \\
\text { Negative }\end{array}$ & $\mathrm{TP}=34$ & $\mathrm{FP}=0$ & $87.20 \%$ & $100.0 \%$ & $100 \%$ & $85.3 \%$ \\
$\begin{array}{c}\text { Extrapulmonary: } \\
\text { Positive }\end{array}$ & $\mathrm{FN}=5$ & $\mathrm{TN}=29$ & & & & \\
Negative & $\mathrm{TP}=7$ & $\mathrm{FP}=0$ & $87.50 \%$ & $100 \%$ & $100 \%$ & $96.0 \%$ \\
\hline
\end{tabular}

PPV : Positive Predictive Value.

NPV : Negative Predictive Value.

Table (3): Comparison of BacT/ALERT MP bottle with Lowenstein Jensen.

\begin{tabular}{|c|c|c|c|c|c|c|}
\hline \multirow[t]{2}{*}{ BacT/ALERT } & \multicolumn{2}{|c|}{$\begin{array}{l}\text { Lowenstein } \\
\text { Jensen }\end{array}$} & \multirow{2}{*}{\multicolumn{2}{|c|}{$\begin{array}{l}\text { Sensi- Speci- } \\
\text { tivity ficity }\end{array}$}} & \multirow[t]{2}{*}{ PPV } & \multirow[t]{2}{*}{ NPV } \\
\hline & Positive & Negative & & & & \\
\hline \multicolumn{7}{|l|}{ Pulmonary: } \\
\hline Positive & $\mathrm{TP}=29$ & $\mathrm{FP}=0$ & $74.4 \%$ & $100 \%$ & $100 \%$ & $74.4 \%$ \\
\hline Negative & $\mathrm{FN}=10$ & $\mathrm{TN}=29$ & & & & \\
\hline \multicolumn{7}{|c|}{ Extrapulmonary: } \\
\hline Positive & $\mathrm{TP}=4$ & $\mathrm{FP}=0$ & $50 \%$ & $100 \%$ & $100 \%$ & $85.7 \%$ \\
\hline Negative & $\mathrm{FN}=4$ & $\mathrm{TN}=24$ & & & & \\
\hline
\end{tabular}

Table (4): Comparison between Lowenstein Jensen culture, Bio FM media and MP BacT/ALERT MP as regard to mean detection time and duration ranges in days.

\begin{tabular}{llccc}
\hline & $\begin{array}{c}\text { LJ } \\
\text { positive }\end{array}$ & $\begin{array}{c}\text { BacT/ALERT MP } \\
\text { positive }\end{array}$ & $\begin{array}{c}\text { Bio FM media } \\
\text { positive }\end{array}$ & $\begin{array}{c}p- \\
\text { value }\end{array}$ \\
\hline Min. & 7 & 7 & 7 & $p^{1} 0.001 * *$ \\
Max. & 56 & 42 & 42 & $p^{2} 0.007 * *$ \\
Mean & 25.06 & 16.33 & 19.07 & $p^{3} 0.205 \mathrm{~ns}$ \\
S.D. & 11.29 & 9.58 & 8.82 & \\
\hline
\end{tabular}

ns : No statistically significant difference $(p>0.05)$.

$* \quad$ : Statistically significant difference $(p<0.05)$.

$* *$ : Statistically high significant difference $(p<0.01)$.

$*_{* *}^{*}$ : Statistically very high significant difference $(p<0.001)$. 
Table (5): Comparison of urine lipoarabinomannan with Lowenstein Jensen.

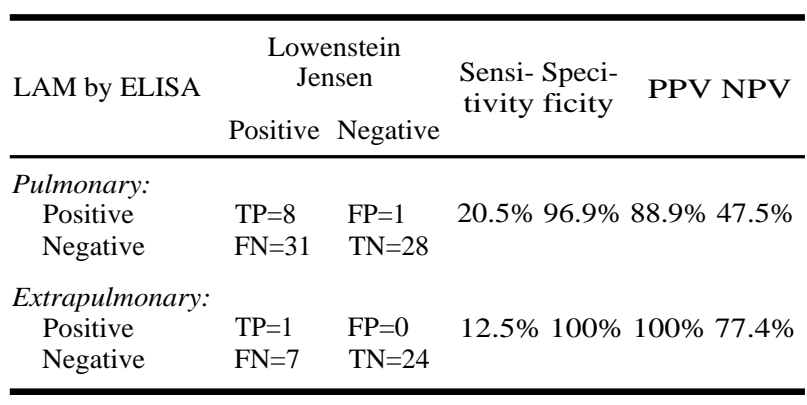

\section{Discussion}

Tuberculosis (TB) represents a global burden and causes significant mortality, mostly in developing countries [10].

Bio-FM medium is also a manual system based on Middlebrook 7H9 broth optimised for mycobacterial growth and containing a coloured indicator that allows the detection of positive cultures which become a dark blue to violet colour [11].

In the present study, in suspected pulmonary tuberculosis cases the Sensitivity and Specificity of Bio-FM were $87.2 \%, 100 \%$ respectively and in suspected extrapulmonary tuberculosis cases were $87.5 \%, 100 \%$ respectively. As regard to the mean detection time and duration ranges in days, here there was statistically significant differences between the LJ positive groups and Bio-FM positive group ( $p=0.007)$.

This results are in agreement with Essa et al., [4] who reported the detection rate $(94 \%$ of the total cases gave positive results on Lowenstein Jensen compared with $92 \%$ on Bio-FM) but the mean detection time of cases on Bio-FM was highly significantly shorter than that of cases on Lowenstein Jensen (12.58 \pm 8.622 days for Bio-FM versus $20.62 \pm 9.640$ days for Lowenstein Jensen $(p<0.001)$, and with Ramarokoto et al., [11] who found no significant difference in detection rate between Lowenstein Jensen compared with that on BioFM. It was $96.58 \%$ and $92.3 \%$ for Lowenstein Jensen and Bio-FM respectively but also the mean detection time of cases on Bio-FM was highly significantly shorter than that of cases on Lowenstein Jensen (12.42 days versus 20.7 days ( $p$ $<0.001)$.

It is possible for all laboratories performing mycobacterial culture to use the Bio FM medium, unlike the other culture systems in liquid medium, it does not require costly specific equipment; the Bio-FM system is entirely manual, with visual reading of cultures, which is therefore simple, although only qualitative, due to its ease of use and the rapid growth time of mycobacteria on Bio FM medium so it can be used for early and rapid detection of M. tuberculosis [11].

Most of automated systems used for TB detection are based on different technologies, such as colorimetric methods that detect bacterial $\mathrm{CO} 2$ production like BacT/ALERT 3D system [5].

In the present study, in suspected pulmonary tuberculosis cases the sensitivity and specificity of BacT/ALERT MP bottle were 74.4\%, 100\% respectively and in suspected extrapulmonary tuberculosis cases were 50\%, 100\% respectively. As regard to the mean detection time and duration, there was statistically significant differences between the LJ positive groups and BacT/ALERT positive group $(p=0.001)$.

This results are in agreement with and with Rahaman et al., [12] who reported that sensitivity and specificity of BacT/ALERT MP culture were $76 \%$ and $85 \%$, and with Naveen and peerapur [13] who reported that the sensitivity, specificity, positive predictive value and the negative predictive value of the $\mathrm{MB} / \mathrm{BACT}$ in comparison to $\mathrm{LJ}$ was $69.5 \%, 94.3 \%, 95 \%$ and $66.7 \%$ respectively and the mean duration of the isolation on $\mathrm{LJ}$ and MB/BACT was 30.81 days and 18.70 days respectively, and with Dionne et al., [14] who reported that sensitivity for mycobacterial recovery was $65 \%$ for the BacT/ALERT MP and recovery time of mycobacterium tuberculosis by BacT/ALERT MP is 16.9 days, and with Parrish et al., [15] reported that sensitivity of Mycobacterium sp. recovery was $66.6 \%$.

Our results are controversary with Amer et al., [16] who reported that BacT/ALERT 3D system show sensitivity $100 \%$ specificity $85.7 \%$ the mean times to detection of mycobacteria by BacT/ALERT 3D system and LJ medium were 14.2 and 24.3 days, respectively and with Sorlozano et al., [17] who reported that sensitivity values for the BacT/ ALERT 3D system range from $78 \%$ to $99 \%$ and with Piersimoni et al., [18] who reported that cultured 67 cases on Lowenstein Jensen in comparison MP/BacT ALERT 3D System, 62 cases gave positive results on Lowenstein Jensen with a detection rate of about $92.53 \%$ and with Parrish et al., [15] reported that the time to detection by the BacT/ ALERT MP system was 25.2 days.

One of the disadvantages of culture in liquid medium is that it does not provide visible colonies increasing the time required for confirmation of 
the result. However, the direct testing of positive BacT/ALERT MP broth medium by PCR allows for the accurate and rapid identification of $\mathrm{M}$. tuberculosis. Especially that ZN staining from the bottle failed to confirm the positive signal in $15.6 \%$ of positive samples. The application of PCR assay directly on positive liquid media of automated systems allows confirmation of the results and fast identification of M. tuberculosis [16].

BacT/ALERT 3D system requires one person who is good at computer basics and is trained, in order to feed the data of the sample and to take the bar code reading. Even though the cost of each $\mathrm{MB} / \mathrm{BACT}$ bottle is costlier as compared to the conventional LJ medium [13].

In the present study, we found that in suspected pulmonary tuberculosis cases, sensitivity and specificity of lipoarabinomannan (LAM by ELISA) were $20.5 \%, 96.9 \%$ respectively and in suspected extrapulmonary tuberculosis cases, sensitivity and specificity were $12.5 \%, 100 \%$ respectively.

These results are in agreement with Daley et al., [19] found that when positivity on either LJ or BACTEC was considered, LAM sensitivity was $17.8 \%$, with a specificity of $87.7 \%$. Compared to positivity on both LJ and BACTEC, LAM sensitivity was $5.8 \%$, with a specificity of $88.8 \%$. Compared to the clinical diagnosis, LAM sensitivity was $20.0 \%$, with a specificity of $83.3 \%$.

Seven studies, assessing test accuracy in microbiologically confirmed cases only, estimates of sensitivity ranged from $13 \%$ to $93 \%$ and specificity from $87 \%$ to $99 \%$. When results were stratified by HIV status in five studies, mean sensitivity in HIVnegative patients was $14 \%$ (range 7-24\%) and in HIV-positive patients 51\% (32-69\%). The sensitivity of the test was 3-53\% higher in HIV-positive than HIV-negative subgroups and was highest with advanced immunosuppression [20].

Our results are controversary with Agha et al., [7] who reported that Urine LAM test had sensitivity, specificity, PPV, NPV, and an accuracy of $81.2 \%, 95.7 \%, 97.2 \%, 73.8 \%$, and $86.4 \%$ respectively. In another study martinson et al., [21] reported that sensitivity and specificity of a positive LAM for culture-confirmed tuberculosis were $65 \%$ and $86 \%$ respectively and with Youssef et al., [22] reported that quantitative urine LAM had a sensitivity of $85.5 \%$, specificity $90 \%$, accuracy $86.1 \%$, positive predictive value $98.1 \%$, and negative predictive value $50 \%$.
The state of circulating LAM may therefore have major implications for urine LAM antigen detection assays, free non-antibody associated LAM is of a size comparable to myoglobin (17 $\mathrm{kd}$ ), which rapidly crosses the glomerular basement membrane. Glomerular filtration of systemically circulating LAM has been the premise to date, on which urine LAM has been interpreted as a correlate of pulmonary TB. However, LAM antigen complexed with IgG (150kd), IgA (370kd) or IgM (1000kd) antibodies would be too large to pass through the normal healthy human glomerulus. Therefore in the presence of circulating anti-LAM immunoglobulin, LAM detected in urine might be more likely to reflect local renal involvement with TB rather than distant pulmonary disease. Increasing senstivity of urine LAM in HIV are due to in ability to produce immunoglobulin such that free circulating LAM can be renally filtered into urine or due to increase risk of disease dissemination resulting in consequent renal involvement [23].

\section{Conclusion:}

Culture procedure is considered to be more sensitive and specific than direct smear examination for the detection of mycobacteria.

- The sensitivity of liquid (Bio FM) media was better than BacT/ALERT MP System.

- Bio FM media and BacT/ALERT MP system show shorter time than Lowenstein Jensen for detection of mycobacterial growth which was very useful to provide faster initiation of treatment and better outcome for the patients.

- Detection of urine lipoarabinomannan by ELISA is insensitive for the diagnosis of $\mathrm{TB}$, although its specificity is adequate.

\section{Conflicts of interest:}

There are no conflicts of interest.

\section{Acknowledgment:}

A great thanks for Faculty of Medicine, Assiut University for its financial support.

Financial support and sponsorship: A grant from the Faculty of Medicine, Assiut University was provided, the grant ID is (1658), for Pro. Dr.: Hebat-Allah G Rashed.

\section{References}

1- WHO: Global tuberculosis report 2015: World Health Organization; 2015.

2- CHAWARE S., INGOLE A., BAHATTARE V. and MANGALKAR S.: Enhancing Sensitivity of Ziehl-Neelsen Stain Using Sodium Hypochlorite Sedimentation Tech- 
nique for Diagnosis of Undiagnosed Cases of Smear Negative Pulmonary Tuberculosis. Indian Journal of Pathology and Oncology, 2 (3): 110-2, 2015.

3- REITHER K., SAATHOFF E., JUNG J., MINJA L.T., KROIDL I., SAAD E., et al.: Low sensitivity of a urine LAM-ELISA in the diagnosis of pulmonary tuberculosis. BMC infectious diseases, 9 (1): 141, 2009.

4- ESSA S.A., ABDEL-SAMEA S.A.R., ISMAEIL Y.M. and MOHAMMAD A.A.: Comparative study between using Lowenstein Jensen and Bio-FM media in identification of Mycobacterium tuberculosis. Egyptian Journal of Chest Diseases and Tuberculosis, 62 (2): 249-55, 2013.

5- MARTINEZ M.R., SARDIÑAS M., GARCIA G., MEDEROS L.M. and DÍAZ R.: Evaluation of BacT/Alert 3D system for Mycobacteria isolates. Journal of Tuberculosis Research, 2 (02): 59, 2014.

6- SHAH M., VARIAVA E., HOLMES C.B., COPPIN A., GOLUB J.E., McCALLUM J., et al.: Diagnostic accuracy of a urine lipoarabinomannan test for tuberculosis in hospitalized patients in a High HIV prevalence setting. Journal of acquired immune deficiency syndromes (1999), 52 (2): 145, 2009.

7- AGHA M.A., EL-HELBAWY R.H., EL-HELBAWY N.G. and EL-SHEAK N.M.: Utility of quantitative analysis of urine lipoarabinomannan in the diagnosis of tuberculosis. Egyptian Journal of Chest Diseases and Tuberculosis, 62 (3): 401-7, 2013.

8- WINN W.C.: Koneman's color atlas and textbook of diagnostic microbiology: Lippincott williams \& wilkins, 2006.

9- SMITHWÌCK R.W.: Laboratory manual for acid-fast microscopy: Public Health Service, Center for Disease Control, Bureau of Laboratories, 1976.

10- GHOLOOBI A., MASOUDI-KAZEMABAD A., MESHKAT M. and MESHKAT Z.: Comparison of culture and PCR methods for diagnosis of Mycobacterium tuberculosis in different clinical specimens. Jundishapur Journal of Microbiology, 7 (2), 2014.

11- RAMAROKOTO H., RASOLONAVALONA T., RATSIMBA L., ANDRIANASOLO D. and RATSITORAHINA M.: Rasolofo Razanamparany V. Evaluation of a rapid culture method on liquid Bio FM (BIO-RAD) medium for the isolation of mycobacteria. The International Journal of Tuberculosis and Lung Disease, 11 (8): 898-903, 2007.

12- RAHAMAN H.S., KUMAR G., MEENA V., AGARWAL A., NATH G. and KUMAR K.: Comparative Evaluation of BacT/ALERT 3D Culture Medium and Nested PCR in the Diagnosis of Tuberculous Meningitis. SAARC Journal of Tuberculosis, Lung Diseases and HIV/AIDS, 11 (2): 15-20, 2015.

13- NAVEEN G. and PEERAPUR B.V.: Comparison of the Lowenstein-Jensen medium, the Middlebrook 7H10 me- dium and MB/BacT for the isolation of Mycobacterium tuberculosis (MTB) from clinical specimens. Journal of clinical and diagnostic research: J.C.D.R., 6 (10): 1704, 2012.

14- DIONNE K., HEDGEPETH A., CARROLL K.C., DICK J.D. and PARRISH1 N.M.: Differences in Time to Detection and Recovery of Mycobacterium tuberculosis between the MGIT 960 and the BacT/Alert MB Automated Culture Systems. American Society for Microbiology, 2007.

15-PARRISH N., DIONNE K., SWEENEY A., HEDGEPETH A. and CARROLL K.: Differences in time to detection and recovery of Mycobacterium spp. between the MGIT 960 and the BacT/ALERT MB automated culture systems. Diagnostic microbiology and infectious disease, 63 (3): 342-5, 2009.

16- AMER S., EL-HEFNAWY A., MAHMOUD M., OKASHA H. and GAD M.: Verification of Bact/Alert 3D System Results for Detection of Mycobacterium Tuberculosis. The Egyptian Journal of Medical Microbiology (EJMM), 25 (4), 2016.

17- SORLOZANO A., SORIA I., ROMAN J., HUERTAS P., SOTO M.J., PIEDROLA G., et al.: Comparative evaluation of three culture methods for the isolation of mycobacteria from clinical samples. J. Microbiol. Biotechnol., 19 (10): 1259-64, 2009.

18- PIERSIMONI C., SCARPARO C., CALLEGARO A., TOSI C.P., NISTA D., BORNIGIA S., et al.: Comparison of MB/BacT ALERT 3D system with radiometric BACTEC system and Löwenstein-Jensen medium for recovery and identification of mycobacteria from clinical specimens: A multicenter study. Journal of clinical microbiology, 39 (2): 651-7, 2001.

19- DALEY P., MICHAEL J., HMAR P., LATHA A., CHORDIA P., MATHAI D., et al.: Blinded evaluation of commercial urinary lipoarabinomannan for active tuberculosis: A pilot study. The International Journal of Tuberculosis and Lung Disease, 13 (8): 989-95, 2009.

20- MINION J., LEUNG E., TALBOT E., DHEDA K., PAI M. and MENZIES D.: Diagnosing tuberculosis with urine lipoarabinomannan: Systematic review and meta-analysis. European Respiratory Journal, 38 (6): 1398-405, 2011.

21- MARTINSON N.A., HOFFMANN C.J. and CHAISSON R.E.: Epidemiology of tuberculosis and HIV: Recent advances in understanding and responses. Proceedings of the American Thoracic Society, 8 (3): 288-93, 2011.

22- YOUSSEF A.A., KAMEL M.H., EISSA H.A., ESSAWY T.S. and MOUSSA H.H.: Evaluation of lipoarabinomannan in the diagnosis of tuberculosis. Egyptian Journal of Bronchology, 10 (3): 291, 2016.

23- WOOD R. and LAWN S.D.: Challenges facing lipoarabinomannan urine antigen tests for diagnosing HIV associated tuberculosis. Expert review of molecular diagnostics, 12 (6): 549-51, 2012. 


\section{طرق محتتلفة فى تشخيص الدرن الرئوى والغير رئوى}

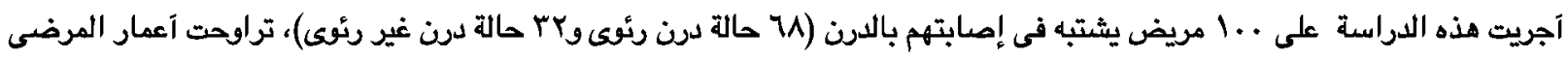

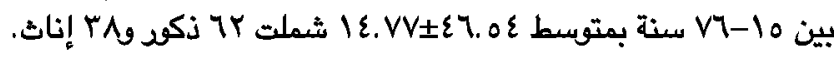

الحالات مقسمة إلى مبحوعتين:

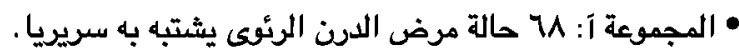

• المجموعة ب: بr حالة مرض الدرن الفير رئوى يشتبه به سريريا.

وقذ خضع جميع الهرضى لما يلى:

• الفحص الميكروسكوبى بإستخدام صبغة الزيل نيلسن.

•زراعة العينات على وسط لوفنثتين جنسن، وسط بيو إف إم وزجاجات الباكت آلرت إم بع.

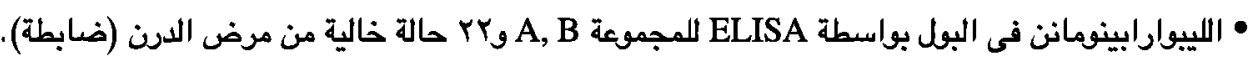

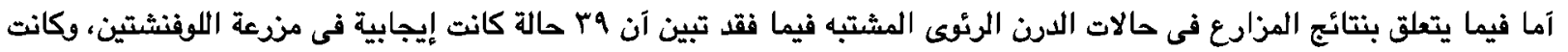

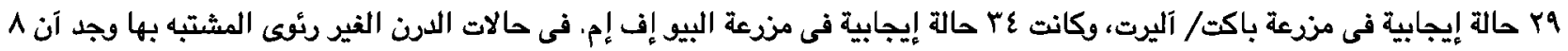

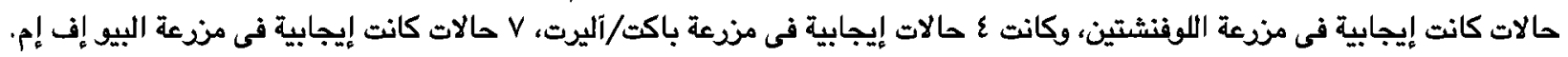

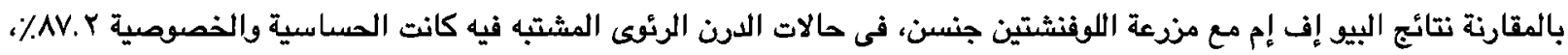

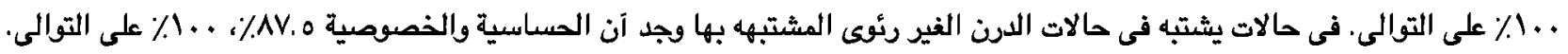

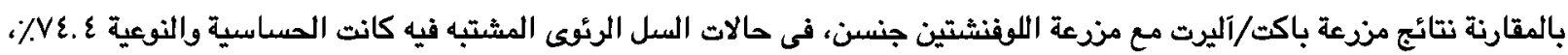

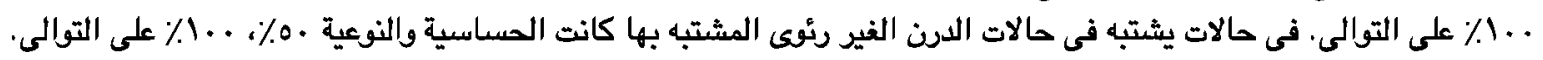

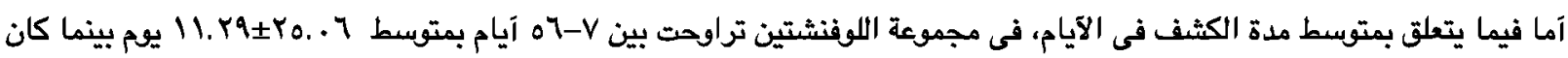

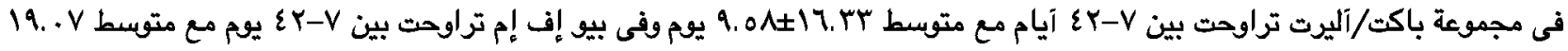
A.AYI

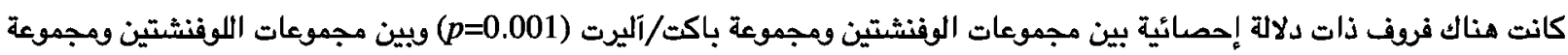

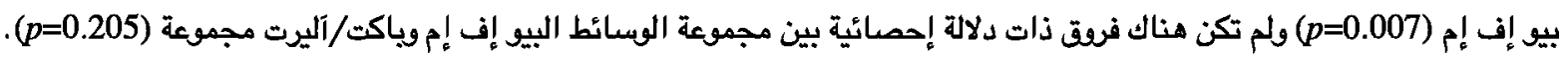
آما فيما يتعلق بنتائج الليبوابينومانان فى حالات الدرن الرئوى المشتبه بها فقد تبين آن 9 حالات كانت إيجابية. فى حالات الدرن الغير رئوى المشتبه بها وجد آن أنعال الئل كانت إيجابية.

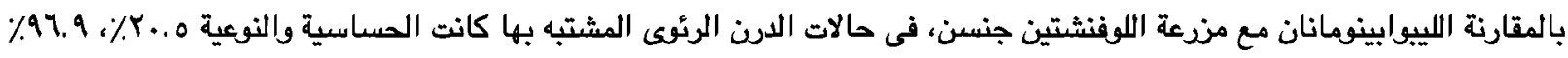

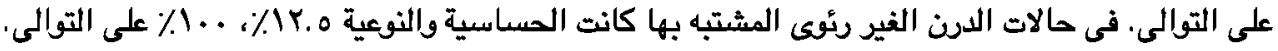

